\title{
Evaluation of masticatory efficiency in maxillofacial trauma patients following occlusal rehabilitation - A clinical study
}

\author{
Suraksha Shrestha ${ }^{1}$ and Santosh Kumar Yadav ${ }^{2 *}$ \\ ${ }^{1}$ Department of Prosthodontics, College of Medical Sciences Teaching Hospital, Bharatpur-10, Chitwan, Nepal \\ ${ }^{2}$ Department of Oral and Maxillofacial Surgery, College of Medical Sciences Teaching Hospital, Bharatpur-10, Chitwan, Nepal
}

\begin{abstract}
Background: Some amount of occlusal discrepancies exist despite of the latest developments in the treatment of fractured jaw bones which may vary from mild to severe which inturn leads to decreased post operative muscle efficiency. Occlusal rehabilitation has been carried out to reduce such discrepancies. This study is aimed to evaluate this post operative muscle efficiency following occlusal rehabilitation with the help of surface electromyography.
\end{abstract}

Materials and methods: Twenty patients who underwent surgical treatment for maxillofacial trauma and required correction for post operative occlusal discrepancies were selected. Pre and post occlusal rehabilitative masticatory efficiency was compared with surface electromyography.

Results: The study revealed a definite increase in masticatory efficiency after post occlusal rehabilitative therapy both in right and left masseter muscle. The mean EMG record values for pre and post occlusal rehabilitation were found to be respectively 826.7 (SD - 179.48) and 1134.01 (SD- 219.02) in the right side, 775.66 (SD- 187.51) and 1067.45 (SD- 197.75) in the left side.

Conclusion: Correction of post operative occlusal discrepancies definitely benefits the patients to exert better masticatory efficiency.

\section{Introduction}

Nothing inspires as humanitarian instincts like trauma. Maxillofacial trauma though is a vast field in itself well studied and researched but still there are certain aspects, which are still in shadows.

In the era prior to the development of craniofacial surgery, the treatment of facial injuries was by necessity, significantly more conservative than it is today. The bones were approached through limited incisions and manipulated into an approximately normal position where they were suspended with wires from the higher, non fractured bony structures. This approach required long periods of immobilization and frequently resulted in unstable results with residual deformity and functional discrepancies like occlusal discrepancies.

Over the years, surgical approach to facial injuries has radically changed. The fractured sites are widely dissected and exposed and under direct vision the bones are replaced in their normal anatomic position, where they are firmly fixed by metal plates and screws. Inspite of all the various principles followed for treatment of fractures like Reduction, Immobilization and Fixation, there might be a result of occlusal discrepancy post operatively due to the following factors like poor patient compliance, inadequate immobilization, poor reduction, delayed healing and rigid fixation.

Hence an adequate post-operative rehabilitation protocol has to be followed to obtain the objectives of reduction and fixation of maxillofacial bone fractures. The protocols being are occlusal rehabilitation, prosthetic rehabilitation, orthodontic rehabilitation and surgical re-correction. The goal of occlusal rehabilitation is to remove the cause of dysfunction and functional restoration of the stomatognathic system.
The purpose of this study was to evaluate the masticatory efficiency in trauma patients, compare the pre-rehabilitation and postrehabilitation masticatory efficiency by evaluating the masseter muscle activity by using surface electromyography and check the efficacy of multidisciplinary approach for the treatment of post-operative occlusal discrepancy.

\section{Materials and methods}

This clinical prospective study was done in Department of Prosthodontics. Twenty patients of age range 16 to 25 years who underwent surgical treatment for maxillofacial trauma and required correction for post-operative occlusal discrepancies were selected.

Armamentarium:

1. Cheek retractor

2. Mouth mirror

3. Vernier caliper

4. Suction tip

5. Articulating paper

6. Electrmyographic apparatus.

Correspondence to: Santosh Kumar Yadav, Department of Oral and Maxillofacial Surgery, College of Medical Sciences Teaching Hospital, Bharatpur-10, P.O. Box-24, Chitwan, Nepal, Email: ssonibpkihs@yahoo.co.in

Key words: electromyography, masticatory efficiency, maxillofacial trauma, occlusal rehabilitation

Received: June 02, 2017; Accepted: June 22, 2017; Published: June 25, 2017 
Following confirmation/detection of an occlusal discrepancy a pre-occlusal rehabilitative masseter muscle activity was recorded using surface electromyography as follows:

Electromyography (EMG) activity was recorded from the superficial masseter muscle bilaterally by means of self adhesive, disposable, pregelled silver chloride surface electrodes. The patient was made to sit comfortably without any head supporting. The patients were instructed to perform maximum voluntary clenching without causing any discomfort and to maintain the same act for atleast one second while the EMG data were recorded (Figure 1). Same procedure was repeated for three times and average reading taken. The digitalized signals were monitored on a computer screen immediately following the test and stored and a mean EMG value for each masseter muscle was calculated.

\section{Results}

Table 1 shows the pre and post occlusal rehabilitation therapy EMG activities recorded from the right and left masseter muscle. Each phase of maximum clenching was recognized as burst of muscle activity. The patient was asked to bring his teeth into occlusion and bite to his maximum force. The masseter muscle activity was recorded. Sets of 3 recordings were made on each side/muscle and average amplitude was taken into considerations. The recordings were compared between the fractured side and non-fractured side before occlusal rehabilitation and after occlusal rehabilitation. This was done on twenty patients who revealed that a definite statistical difference between EMG recordings of the fractured and non-fractured side, and upon correction of occlusion and collateral physiotherapy, there was a definite masseter muscle activity increased on the fractured side which in turn would increase or aid in the improvement of masticatory efficiency. The mean values are graphically represented in Figure 2.

The EMG values of masseter muscle were used as a measure of muscle efficiency in this study as done by Steven J. Lindauer [1].

\section{Discussion}

Electromyographic systems measure the electrical potential of muscle units. Recordings from such systems have been used to evaluate muscle activity during mastication and command mandibular movements.

The masseter muscle has usually been selected for study although the other muscles of mastication have occasionally been used. The probable reason for a decrease in bite force after treatment of maxillofacial

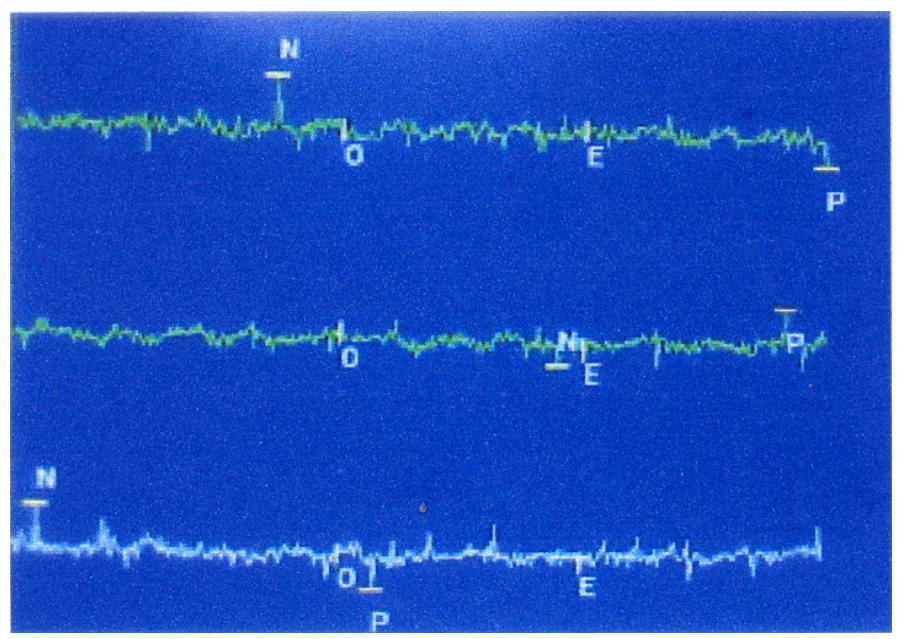

Figure1. Electromyography graph showing masseter muscle activity.

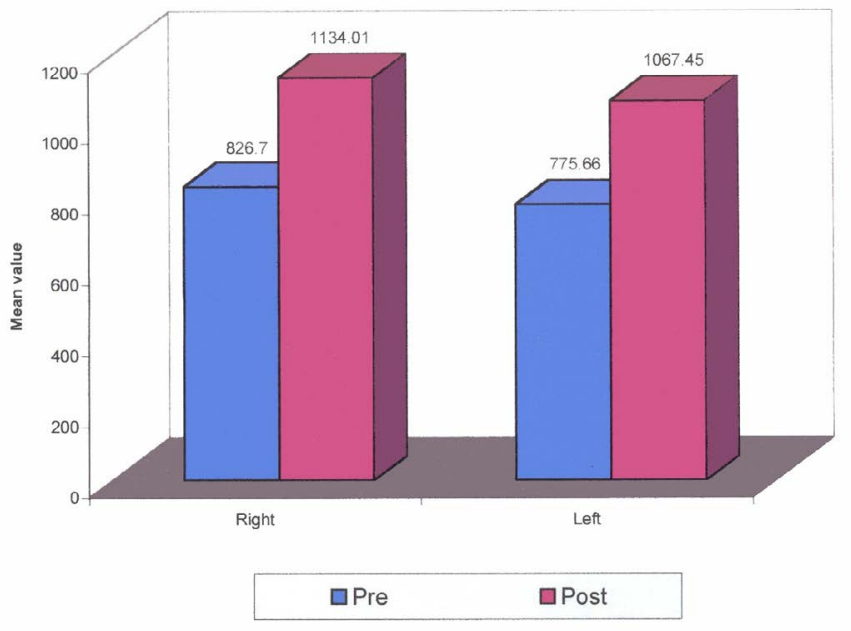

Table 1. Electromyography recording values from right and left masseter muscle.

\begin{tabular}{|c|c|c|}
\hline Side & Pre & Post \\
\hline RightMean & 826.7225 & 1134.0100 \\
Standard deviation & 179.4871 & 219.0256 \\
Median & 829.4350 & 1124.3400 \\
Minimum & 516.83 & 822.27 \\
Maximum & 1177.19 & 1562.09 \\
\hline LeftMean & 775.6650 & 1067.4535 \\
Standard deviation & 187.5155 & 197.7591 \\
Median & 718.3300 & 1015.1350 \\
Minimum & 527.86 & 842.22 \\
Maximum & 1123.31 & 1428.84 \\
\hline TotalMean & 801.1938 & 1100.7318 \\
Standard deviation & 183.0122 & 208.7100 \\
Median & 817.9050 & 1054.6550 \\
Minimum & 516.83 & 822.27 \\
Maximum & 1177.19 & 1562.09 \\
\hline
\end{tabular}

bone fractures is traumatic and surgical damage to the masseter and temporalis muscles. Placing fixation hardware from an intra-oral approach necessitates the masseter muscle being stripped from their attachments. Further, a transfacial trochar used for instrumentation may also damage the masseter muscle. Hence, masseter muscle was taken into consideration for EMG studies. Lower bite forces occurred in patients with occlusal dysfunction in their masticatory system when compared with healthy people [2] and the bite force increased as the symptoms of masticatory dysfunction decreased [3].

Size and mechanical advantage of the jaw adductor muscles, sensitivity of the teeth, muscles and TMJ and the patient's willingness to exert maximum effort have been found to influence generation of maximum occlusal force.

Attempts have been made to measure masticatory force either directly, using various types of transducers in the mouth or indirectly from muscle activity levels. Ahlgren J et al. [4] and William R. Profit et al. [5] said that Electromyography can be a reliable index to estimate masticatory force and estimates of masticatory force was based on an indirect method using EMG levels during maximum voluntary clenching act which was used by Bakke $\mathrm{M}$ et al. [6]. A more reliable estimate of masticatory force is achieved by averaging the EMG values [7]. There were significant differences found between EMG mean values recorded on operated side before and after occlusal rehabilitation that corresponded to the result of the study by EVA Helkimo et al. [3]. 
The values for maximum biting force reported in this study compares favorably to those reported in the past by Linderholm and Wennstrom [8].

Limitations of the study:

1. The decreased pain threshold levels associated with the process of arch bar application before occlusal rehabilitation could seriously impair the ability or desire to bite hard into occlusion with normal forces [1].

2. With different positions of the mandible, the effect of gravity is altered causing activation of different motor units resulting various amplitude and sequence of onset of the contractile pattern [9].

3. Changes in skin resistance might be responsible for variation in the parameters of muscle activity [10].

4. Movement of the mandible in various direction and speeds during masticatory activities alters motor unit activity of the muscle and vary the sequence contracting of muscle fibers [11].

5. A difference in body build and general muscular strength certainly would contribute to maximum occlusal force differences [5].

\section{Conclusions}

Within these limitations of the study, following conclusions could be drawn:

1. There is decrease in the masseter muscle activity in the postoperative period.

2. Statistically significant increase in the masseter muscle activity is found after occlusal rehabilitation and co-lateral physiotherapy.
3. Multidisciplinary approach has proved beneficial in increasing the masseter muscle activity in post rehabilitation patients and results are encouraging.

\section{References}

1. Steven J (1994) Lindauer. discussion: estimated masticatory forces in patients before orthognathic surgery. J Oral and Maxillofac Surg 52: 136-137.

2. Molin C (1972) Vertical isometric muscle forces of the mandible. A comparative study of subjects with and without manifest mandibular pain dysfunction syndrome. Acta Odontol Scand 30: 485-499.[Crossref]

3. Helkimo E, Carlsson GE, Carmeli Y (1975) Bite force in patients with functional disturbances of the masticatory system. J Oral Rehabil 2: 397-406.[Crossref]

4. Ahlgren J, Owall B (1970) Muscular activity and chewing force: a polygraphic study of human mandibular movements. Arch Oral Biol 15: 271-280.[Crossref]

5. Proffit WR, Fields HW, Nixon WL (1983) Occlusal forces in normal- and long-face adults. $J$ Dent Res 62: 566-570.[Crossref]

6. Bakke M, Paulsen HU (1989) Herbst treatment in late adolescence: Clinical, electromyographic, kinesiographic, and radiographic analysis of one case. Eur $J$ Orthodont 11: 397-407.[Crossref]

7. Neill DJ, Kydd WL, Nairn RI, Wilson J (1989) Functional loading of the dentition during mastication. J Prosthet Dent 62: 218-228.[Crossref]

8. Linderholm H, Wennström A (1970) Isometric bite force and its relation to general muscle forge and body build. Acta Odontol Scand 28: 679-689.[Crossref]

9. Brennan, Amsterdam (1963) Postural effects of occlusion. Dental Prog 4: 43-47.

10. Mohller E (1966) The chewing apparatus. Acta Physiolo Scand 69: 280.

11. Ahlgren J (1967) Kinesiology of the mandible. An EMG study. Acta Odontol Scand 25: 593-611.[Crossref]

Copyright: (C2017 Shrestha S. This is an open-access article distributed under the terms of the Creative Commons Attribution License, which permits unrestricted use, distribution, and reproduction in any medium, provided the original author and source are credited. 